\title{
Theory and Design of a Cavity Attenuator
}

\author{
By J. J. Freeman
}

\begin{abstract}
The fields generated by an arbitrary current distribution exciting a piston-type or cavity attenuator are developed, and symmetric distributions exciting maximum amplitudes of the dominant mode and minimum amplitudes of unwanted modes are investigated. The relative error in voltage measurement due to spurious modes is computed as a function of spacing between exciting and receiving coils for certain simple current distributions. The relative merits of circular and rectangular attenuator cross sections are discussed.
\end{abstract}

\section{Introduction}

The cavity attenuator, also known as a piston or mutual inductance type attenuator, was first designed by Wheeler, Harnett, and Case [1] ${ }^{1}$ for use in signal generators, and has since found wide application. It consists of a hollow tube excited below its cut-off frequency and a coil or capacitor, which picks up the attenuated field. As the generated electromagnetic field falls off exponentially with distance from the exciting source, and as the attenuation constant may be computed from the dimensions of the tube, the ratio of any two voltages is reduced to a measurement of length.

Preparatory to the design and construction of such an attenuator, it was thought advisable to investigate the theory to determine the kind of excitation required for optimum mode purity, and an evaluation of the amplitudes of the unwanted modes, for different types of excitation. A rigorous solution of the problem is impossible, but an approximate solution that is useful consists in determining the field produced by an arbitrarycurrent distribution within a waveguide closed at both ends. Here the perturbation of the field by the receiving loop is neglected. It may also be assumed that the exciting current distribution is maintained by a constant-current generator.

Thus, given a distribution of current density, $\mathbf{J}\left(x^{\prime}, y^{\prime}, z^{\prime}\right) e^{-j \omega t}$, the field produced within a perfectly conducting cavity is sought. The scalar analogue, where $\mathbf{J}\left(x^{\prime}, y^{\prime}, z^{\prime}\right)$ is replaced by $\rho$, the charge density, is well known, and has been treated elegantly by Sommerfeld [2]. The vector problem

\footnotetext{
${ }^{1}$ Figures in brackets indicate the literature references at the end of this paper.
}

is solved analogously, by expansion into orthogonal vector wave functions, and has been treated by Heitler [3], Condon [4], and others. For completeness, the method is outlined below.

\section{Expansion of the Field into Normal Vector Functions}

MKS units are used and the fields and source are assumed to vary as $e^{-j_{\omega} t}$. Then, according to Maxwell's equations [5], one must find a solution for

$$
\nabla \times \nabla \times \mathbf{E}-k^{2} \mathbf{E}=j \omega \mu \mathbf{J},
$$

subject to the boundary condition that $\mathbf{n} \times \mathbf{E}=0$ on the surface $S$ of the cavity, where $\mathbf{n}$ is the normal on $S . \mathbf{E}$ is the electric field, $\mathbf{J}$ the impressed current density, and $k^{2}=\omega^{2} \mu \epsilon$. As any vector field may be shown to consist of two parts, one of zero curl, and the other of zero divergence, let $\mathbf{J}=\mathbf{J}_{1}+\mathbf{J}_{2}$, where curl $\mathbf{J}_{1}=0$, and div. $\mathbf{J}_{2}=0$.

Let $\mathbf{E}=\mathbf{E}_{1}+\mathbf{E}_{2}$, where curl $\mathbf{E}_{1}=0$, and $\operatorname{div} \mathbf{E}_{2}=0$. Then, if

and if

$$
\operatorname{curl}^{2} \mathbf{E}_{2}-k^{2} \mathbf{E}_{2}=j \omega \mu \mathbf{J}_{2}
$$

$$
\mathbf{E}_{1}=-\frac{j \omega \mu}{k^{2}} \mathbf{J}_{1},
$$

then $\mathbf{E}=\mathbf{E}_{1}+\mathbf{E}_{2}$ satisfies eq 1 .

If $\psi_{\alpha}$ is a solution of $\nabla^{2} \dot{\psi}_{\alpha}+k^{2}{ }_{\alpha} \psi_{\alpha}=0$, and $\psi_{\alpha}=0$ on $S$, the interior surface of the cavity, $\nabla \psi_{\alpha} \times \mathbf{n}=0$ on $S$. If the $\psi_{\alpha}$ are normalized and orthogonal, then $\frac{1}{k_{\alpha}} \nabla \psi_{\alpha}$ are normalized and orthogonal, because

$$
\begin{aligned}
& \int \nabla \psi_{\alpha} \cdot \nabla \psi_{\beta} d \tau=\mathcal{S}\left(\nabla \cdot\left[\psi_{\beta} \nabla \psi_{\alpha}\right]-\psi_{\beta} \nabla^{2} \psi_{\alpha}\right) d \tau= \\
& k_{\alpha}{ }^{2} \int \psi_{\alpha} \psi_{\beta} d \tau
\end{aligned}
$$


Here $d \tau$ is an element of volume, and the integration is performed throughout the volume of the cavity.

Then if we call the normalized $\frac{1}{k_{\alpha}} \nabla \psi_{\alpha}=\mathbf{E}_{\alpha}$, an arbitrary irrotational vector function whose tangential component vanishes on $S$ may be developed in terms of the $\mathbf{E}_{\alpha}$.

Thus,

where

$$
\mathbf{E}_{1}=\sum_{\alpha} \alpha_{\alpha} \mathbf{E}_{\alpha}
$$

$$
\begin{gathered}
\alpha_{\alpha}=\int \mathbf{E}_{1} \cdot \mathbf{E}_{\alpha} d \tau \\
\mathbf{J}_{1}=\sum_{\alpha} \int \mathbf{J}_{1} \cdot \mathbf{E}_{\alpha} d \tau \mathbf{E}_{\alpha}
\end{gathered}
$$

Substituting the above expression for $\mathbf{J}_{1}$ in eq 3 ,

$$
\mathbf{E}_{1}(r)=-\frac{j \omega \mu}{k^{2}} \sum_{\alpha} \int \mathbf{J}_{1}\left(r^{\prime}\right) \cdot \mathbf{E}_{\alpha}\left(r^{\prime}\right) d \tau^{\prime} \mathbf{E}_{\alpha}(r) .
$$

If one had a complete set of orthogonal vector wave functions $\mathbf{E}_{\beta}$, and if $\operatorname{div} \mathbf{E}_{\beta}=0$, and $\mathbf{n} \times \mathbf{E}_{\beta}=0$, an arbitrary solenoidal vector, whose tangential component vanished on $S$, in particular $\mathbf{E}_{2}$, could be expanded in terms of the $\mathbf{E}_{\beta}$. Suppose the $\mathbf{E}_{\beta}$ are solutions of

$$
\operatorname{curl}^{2} \mathbf{E}_{\beta}-k_{\beta}^{2} \mathbf{E}_{\beta}=0,
$$

where $\mathbf{n} \times \mathbf{E}_{\beta}=0$, and $\operatorname{div} \mathbf{E}_{\beta}=0$. Then it may be shown that the $\mathbf{E}_{\beta}$ are orthogonal to each other, and to the $\mathbf{E}_{\alpha}$.

Let $\mathbf{E}_{2}=\Sigma e_{\beta} \mathbf{E}_{\beta}$, and let $\mathbf{J}_{2}=\Sigma j_{\beta} \mathbf{E}_{\beta}$. Then $j_{\beta}=\int \mathbf{J}_{2} \cdot \mathbf{E}_{\beta} d \tau$. Substituting the above values for $\mathbf{E}_{2}$ and $\mathbf{J}_{2}$ in eq 2 ,

$$
\left.\begin{array}{l}
e_{\beta}=\frac{j \omega \mu}{k_{\beta}^{2}-k^{2}} \boldsymbol{\int} \mathbf{J}_{2} \cdot \mathbf{E}_{\beta} d \tau \\
\mathbf{E}_{2}=j \omega \mu \sum_{\boldsymbol{\beta}} \frac{\boldsymbol{\int} \mathbf{J}_{2}\left(r^{\prime}\right) \cdot \mathbf{E}_{\beta}\left(r^{\prime}\right)}{\left(k_{\boldsymbol{\beta}}^{2}-k^{2}\right)} d \tau^{\prime} \mathbf{E}_{\beta}(r)
\end{array}\right\}
$$

Since the $\mathbf{E}_{\alpha}$ are orthogonal to the $\mathbf{E}_{\beta}$, and the $\mathbf{E}_{\alpha}$ and $\mathbf{E}_{\beta}$ are assumed complete for expansions of electric fields of zero curl and zero divergence, respectively, $\int \mathbf{J}_{2} \cdot \mathbf{E}_{\alpha} d \tau=\int \mathbf{J}_{1} \cdot \mathbf{E}_{\beta} d \tau=0$. Accordingly, we may drop the subscripts in $\mathbf{J}_{1}$ and $\mathbf{J}_{2}$ in eq 4 and 5 , and substitute the total current density, J. Thus

$$
\left.\begin{array}{l}
\mathbf{E}_{1}(r)=\frac{-j \omega \mu}{k^{2}} \sum \boldsymbol{J} \mathbf{J}\left(r^{\prime}\right) \cdot \mathbf{E}_{\alpha}\left(r^{\prime}\right) d \tau^{\prime} \mathbf{E}_{\alpha}(r) \\
\mathbf{E}_{2}(r)=j \omega \mu \sum \frac{\boldsymbol{S} \mathbf{J}\left(r^{\prime}\right) \cdot \mathbf{E}_{\beta}\left(r^{\prime}\right) d \tau^{\prime}}{\left(k_{B}^{2}-k^{2}\right)} \mathbf{E}_{\beta}(r)
\end{array}\right\}
$$

\section{Attenuators of Circular Cross Section}

The normal vector modes are now considered for the circular cylinder of radius $a$, and length $d$, where $\mathbf{i}_{1}, \mathbf{i}_{2}$ and $\mathbf{k}$ are the unit vectors in the directions of increasing $r, \theta$ and $z$.

Orthogonal vector wave functions have been treated by Stratton [6], and the normal vector modes are most easily obtained by following his treatment, and satisfying the appropriate boundary conditions. For the interior of a circular cylinder,

$$
\begin{aligned}
& \mathbf{E} \alpha=\mathbf{E}_{o m l}^{e}=\frac{F_{n m}}{\sqrt{\lambda^{2}{ }_{n m}+\frac{l^{2} \pi^{2}}{d^{2}}}} \\
& {\left[\left\{\lambda_{n m} J_{n}^{\prime}\left(\lambda_{n m} r\right)\left(\begin{array}{c}
\cos n \theta \\
\sin n \theta
\end{array}\right) \mathbf{i}_{1}+\right.\right.} \\
& \left.\frac{n}{r}\left(\begin{array}{r}
-\sin n \theta \\
\cos n \theta
\end{array}\right) J_{n}\left(\lambda_{n m} r\right) \boldsymbol{i}_{2}\right\} \sin \frac{l \pi z}{d}+ \\
& \left.\left(\begin{array}{c}
\cos n \theta \\
\sin n \theta
\end{array}\right) J_{n}\left(\lambda_{n m} r\right) \frac{l_{\pi}}{d} \cos \frac{l \pi z}{d} \mathbf{k}\right]
\end{aligned}
$$

Here,

$$
\lambda_{n m}=\frac{u_{n m}}{a}, \text { where } J_{n}\left(u_{n m}\right)=0
$$

and

$$
k_{\alpha}^{2}=k_{n m l}^{2}=\lambda_{n m}^{2}+\left(\frac{l \pi}{d}\right)^{2}
$$

$J_{n}$ is the $n$th order Bessel function. The normalizing factor, $F_{n m}$ is

$$
F_{\substack{n m \\
o}}^{e}=\left(\begin{array}{c}
\sqrt{4-2 \delta_{0}^{n}} \\
\sqrt{4-4 \delta_{0}^{n}}
\end{array}\right) \frac{1}{a \sqrt{\pi d}\left|J_{n-1}\left(\lambda_{n m} a\right)\right|},
$$

where $\delta_{0}^{n}=0$, for $n \neq 0$, and $\delta_{0}^{n}=1$, for $n=0$

The normal vector modes $\mathbf{E}_{\beta}$, consist of two independent, mutually orthogonal sets, the transverse electric type, which we designate as $\mathbf{M}_{\beta}$, and the transverse magnetic type, which we designate as $\mathbf{N}_{6}$.

$$
\mathbf{M}_{\beta}=\mathbf{M}_{\substack{n m \\
o}}^{e}=A_{\substack{n m \\
o}}^{e}\left[\frac{n}{r}\left(\begin{array}{r}
-\sin n \theta \\
\cos n \theta
\end{array}\right) J_{n}\left(\delta_{n m} r\right) \mathbf{i}_{1}-\delta_{n m} J_{n}^{\prime}\left(\delta_{n m} r\right)\left(\begin{array}{c}
\cos n \theta \\
\sin n \theta
\end{array}\right) \mathbf{i}_{2}\right] \times \sin \frac{l \pi z}{d}
$$


Here,

$$
\delta_{n m}=\frac{v_{n m}}{a}, J_{n}^{\prime}\left(v_{n m}\right)=0
$$

and

$$
k_{n m l}^{2}=\delta_{n m}^{2}+\left(\frac{(l \pi)}{d}\right)^{2}
$$

$$
\begin{gathered}
\therefore_{\beta}=N_{c^{\prime} m}=\frac{F_{n m}^{e}}{\lambda_{n m}} \sqrt{\frac{o}{\lambda_{n m}^{2}+\frac{l^{2} \pi^{2}}{d^{2}} \sqrt{1+\delta_{0}^{l}}}}\left[-\frac{l \pi}{d} \sin \frac{l \pi z}{d}\left\{\left(\begin{array}{c}
\cos n \theta \\
\sin n \theta
\end{array}\right) \lambda J_{n}^{\prime}\left(\lambda_{n m} r\right) \mathbf{i}_{1}+\frac{n}{r}\left(\begin{array}{r}
-\sin n \theta \\
\cos n \theta
\end{array}\right) J_{n}\left(\lambda_{n m} r\right) \mathbf{i}_{2}+\right.\right. \\
\left.\lambda_{n m}^{c} \cos \frac{l \pi z}{d} J_{n}\left(\lambda_{n m} r\right)\left(\begin{array}{c}
\cos n \theta \\
\sin n \theta
\end{array}\right) \mathbf{k}\right]
\end{gathered}
$$

When no ambiguity results, the subscripts $l, m$, $n$ are omitted and $\lambda_{n m}$, is written, for instance, as $\lambda$. The even, odd designations $\left(\begin{array}{l}0 \\ e\end{array}\right)$, are also omitted and one writes $\mathbf{m}_{o}^{e}$ as $\mathbf{m}$, the even, odd designation being implied.

Substituting eq 11 and 13 in eq 6 ,

$$
\begin{gathered}
\mathbf{E}_{2}=j \omega \mu \sum_{n, m, l} \frac{\int \mathbf{J}\left(r^{\prime}\right) \cdot M_{0}^{e}\left(r^{\prime}\right) d \tau^{\prime} M_{0}^{e}(r)}{\delta^{2}+\left(\frac{1 \pi}{d}\right)^{2}-k^{2}}+ \\
j \omega \mu \sum_{n, m, l} \frac{\int \mathbf{J}\left(r^{\prime}\right) \cdot \mathbf{N}_{0}^{e}\left(r^{\prime}\right) d \tau^{\prime} \mathbf{N}_{0}^{e}(r) .}{\lambda^{2}+\left(\frac{l \pi}{d}\right)^{2}-k^{2}}
\end{gathered}
$$

Call the first summation $\mathbf{A}$, the second that

$$
\mathbf{E}_{2}=\mathbf{A}+\mathbf{B} \text {. }
$$

$$
\text { Let } \mathbf{M}_{0 m l}^{e}=A_{n m} \mathbf{m}_{n m}^{e} \sin \frac{l \pi z}{d}
$$

where

$$
\mathbf{m}_{n m}^{e}=\frac{n}{r}\left(\begin{array}{r}
-\sin n \theta \\
\cos n \theta
\end{array}\right) J_{n}(\delta r) \mathbf{i}_{1}-\left(\begin{array}{c}
\cos n \theta \\
\sin n \theta
\end{array}\right) \delta J_{n}^{\prime}(\delta r) \mathbf{i}_{2}
$$

Then

$$
\mathbf{A}=j \omega \mu \sum_{n, m} A_{n m}^{2} \int \mathbf{J}\left(r^{\prime}\right) \cdot \mathbf{m}\left(r^{\prime}\right) \mathbf{m}(r) \sum_{l=1}^{\infty} \frac{\sin \frac{l \pi z^{\prime}}{d} \sin \frac{l \pi z}{d}}{\delta^{2}-k^{2}+\left(\frac{(l \pi)}{d}\right)^{2}} d \tau^{\prime}
$$

In appendix I, it is shown that

$$
\begin{aligned}
& \sum_{l=1}^{\infty} \frac{\sin \frac{l \pi z^{\prime}}{d} \sin \frac{l \pi z}{d}}{\delta^{2}-k^{2}+\left(\frac{(1 \pi)}{d}\right)^{2}}=S_{1}= \\
& \frac{d}{2 \gamma} \sinh \gamma z^{\prime}\left(\frac{1-e^{-2 \gamma(d-z)}}{1-e^{-2 \gamma d}}\right) e^{-\gamma z},
\end{aligned}
$$

where $\gamma_{n m}=\sqrt{\frac{v_{n m}^{2}-k^{2}}{a^{2}}}$
Substituting the above in eq 15 , and substituting the value of $A_{n m}$ given in eq $12 a$.

$$
\boldsymbol{A}=j \omega \mu \sum_{n=0}^{\infty} \sum_{m=1}^{\infty} \frac{2 e^{-\gamma^{2}}\left(\frac{1-e^{-2 \gamma(d-z)}}{1-e^{-2} \gamma^{\bar{d}}}\right)}{\pi\left(1+\delta_{0}^{n}\right)\left(v_{n m}^{2}-n^{2}\right) J_{n}^{2}\left(v_{n m}\right) \gamma}
$$

Let

$$
\int \mathbf{J}\left(r^{\prime}\right) \cdot \mathbf{m}\left(r^{\prime}\right) \mathbf{m}(r) \sinh \gamma z^{\prime} d \tau^{\prime}
$$

$$
\begin{aligned}
\mathbf{n}_{0}^{e}= & {\left[-\lambda_{n m} J_{n}^{\prime}(\lambda r)\left(\begin{array}{c}
\cos \mathrm{n} \theta \\
\sin \mathrm{n} \theta
\end{array}\right) \mathbf{i}_{1}+\right.} \\
& \left.\left(\begin{array}{r}
\sin n \theta \\
-\cos n \theta
\end{array}\right) \frac{n}{r} J_{n}(\lambda r) \mathbf{i}_{2}\right]
\end{aligned}
$$

and

$$
\mathbf{p}_{0}^{e}=\left(\begin{array}{c}
\cos n \theta \\
\sin n \theta
\end{array}\right) J_{n}(\lambda r) \mathbf{k}
$$

Substituting eq 17 and eq 18 in the second sum of eq14, we get

$$
\begin{aligned}
\mathbf{B}= & j \omega \mu \boldsymbol{J} \mathbf{J}\left(r^{\prime}\right) \cdot \sum_{n, m} \frac{F_{n, m}^{e}}{\lambda^{2}} \\
& \left\{\mathbf{n}\left(r^{\prime}\right) \mathbf{n}(r) \sum_{l=1}^{\infty} \frac{\left(\frac{l \pi}{d}\right)^{2} \sin \frac{l \pi z^{\prime}}{d} \sin \frac{l \pi z}{d}}{\left(\lambda^{2}+\frac{l^{2} \pi^{2}}{d^{2}}\right)\left(\lambda^{2}-k^{2}+\frac{l^{2} \pi^{2}}{d^{2}}\right)}+\right. \\
& \lambda^{2} \mathbf{n}\left(r^{\prime}\right) \mathbf{p}(r) \sum_{l=1}^{\infty} \frac{\frac{l \pi}{d} \sin \frac{l \pi z^{\prime}}{d} \cos \frac{l \pi z}{d}}{\left(\lambda^{2}+\frac{l^{2} \pi^{2}}{d^{2}}\right)\left(\lambda^{2}-k^{2}+\frac{l^{2} \pi^{2}}{d^{2}}\right)}+ \\
& \lambda^{2} \mathbf{p}\left(r^{\prime}\right) \mathbf{n}(r) \sum_{l=1}^{\infty} \frac{\frac{l \pi}{d} \cos \frac{l \pi z^{\prime}}{d} \sin \frac{l \pi z}{d}}{\left(\lambda^{2}+\frac{l^{2} \pi^{2}}{d^{2}}\right)\left(\lambda^{2}-k^{2}+\frac{l^{2} \pi^{2}}{d^{2}}\right)}+ \\
& \lambda^{4} \mathbf{p}\left(r^{\prime}\right) \mathbf{p}(r) \\
& \left.\sum_{l=0}^{\infty} \frac{\cos \frac{l \pi z^{\prime}}{d} \cos \frac{l \pi z}{d}}{\left(\lambda^{2}+\frac{l^{2} \pi^{2}}{d^{2}}\right)\left(1+\delta_{0}^{l}\right)\left(\lambda^{2}-k^{2}+\frac{l^{2} \pi^{2}}{d^{2}}\right)}\right\} d \tau^{\prime}
\end{aligned}
$$


Similarly, if eq 17,18 , and 7 are substituted in eq 6 ,

$$
\begin{aligned}
& \mathbf{E}_{1}=-\frac{j \omega \mu}{k^{2}} \boldsymbol{S} \mathbf{J}\left(r^{\prime}\right) \cdot \sum_{n, m} F_{n m}^{2} \\
& \left\{\mathbf{n}\left(r^{\prime}\right) \mathbf{n}(r) \sum_{l=1}^{\infty} \frac{\sin \frac{l \pi z^{\prime}}{d} \sin \frac{l \pi z}{d}}{\lambda^{2}+\frac{l^{2} \pi^{2}}{d^{2}}}-\mathbf{n}\left(r^{\prime}\right) \mathbf{p}(r)\right. \\
& \sum_{l=1}^{\infty} \frac{\frac{l \pi}{d} \sin \frac{l \pi z^{\prime}}{d} \cos \frac{l \pi z}{d}}{\lambda^{2}+\frac{l^{2} \pi^{2}}{d^{2}}}-\mathbf{p}\left(r^{\prime}\right) \mathbf{n}(r) \\
& \sum_{l=1}^{\infty} \frac{\frac{l \pi}{d} \cos \frac{l \pi z^{\prime}}{d} \sin \frac{l \pi z}{d}}{\lambda^{2}+\frac{l^{2} \pi^{2}}{d^{2}}}+\mathbf{p}\left(r^{\prime}\right) \mathbf{p}(r) \\
& \sum_{l=1}^{\infty} \frac{\frac{l^{2} \pi^{2}}{d^{2}} \cos \frac{l \pi z^{\prime}}{d} \cos \frac{l \pi z}{d}}{\lambda^{2}+\frac{l^{2} \pi^{2}}{d^{2}}} \mid d \tau^{\prime}
\end{aligned}
$$

With a little manipulation, $\mathbf{E}_{1}$ and $\mathbf{B}$ may be combined to give

$$
\begin{aligned}
& \mathbf{E}_{1}+\mathbf{B}=\frac{j}{\omega \epsilon} \int \mathbf{J}\left(r^{\prime}\right) \cdot \sum_{n, m} F^{2} \\
& \left\{\mathbf{n}\left(r^{\prime}\right) \mathbf{n}(\mathbf{r}) \frac{k^{2}-\lambda^{2}}{\lambda^{2}} \sum_{l=1}^{\infty} \frac{\sin \frac{l \pi z^{\prime}}{d} \sin \frac{l \pi z}{d}}{\lambda^{2}-k^{2}+\frac{l^{2} \pi^{2}}{d^{2}}}+\right. \\
& \mathbf{E}_{T E}=\mathbf{A}=j \omega \mu \sum_{n=0}^{\infty} \sum_{m=1}^{\infty} \frac{2 e^{-\gamma}{ }_{n m_{0}}^{z}\left(1-e^{-2 \gamma} n{ }^{(d-z)}\right)}{\pi\left(1+\delta_{0}^{n}\right)\left(v_{n m}^{2}-n^{2}\right) \boldsymbol{J}_{n}^{2}\left(v_{n m}\right) \gamma_{n m}\left(1-e^{-2 \gamma}{ }_{n m}{ }^{d}\right)}\left[\boldsymbol{S} \mathbf{J}\left(r^{\prime}\right) \cdot \mathbf{m}_{o}^{e}\left(r^{\prime}\right) \mathbf{m}_{o}^{o}(r) \sin h \gamma_{n m} z^{\prime} d \tau^{\prime}\right] \\
& \mathbf{E}_{T M}=\mathbf{E}_{1}+\mathbf{B}=-\frac{j}{\omega \epsilon} \sum_{n=0}^{\infty} \sum_{m=1}^{\infty} \frac{\left(2-\delta_{0}^{n}\right) e^{-\eta} n m^{z^{\prime}}}{\pi u_{n m}^{2} J_{n-1}^{2}\left(u_{n m}\right)\left(1-e^{-2 \eta}{ }_{n m}{ }^{d}\right)}\left[\left(1-e^{-2 \eta}{ }_{n m}{ }^{(d-z)}\right) \boldsymbol{J} \mathbf{J}\left(r^{\prime}\right) .\right. \\
& {\left[\mathbf{n}_{n m}\left(\mathbf{r}^{\prime}\right) \mathbf{n}_{n m}(\mathbf{r}) \eta_{n m} \sin h \eta_{n m} z^{\prime}-\lambda_{n m}^{2} \mathbf{p}_{n m}\left(r^{\prime}\right) \mathbf{n}_{n m}(r) \cos h \eta_{n m} z^{\prime}\right] d \tau^{\prime}+\left(1+e^{-2 \eta}{ }_{n m}{ }^{(d-z)}\right) \boldsymbol{S} \mathbf{J}\left(r^{\prime}\right) .} \\
& \left.\left[\lambda_{n m}^{2} n_{n m}\left(r^{\prime}\right) \mathbf{p}(r) \sin h \eta_{n m} z^{\prime}-\lambda_{n m}^{4} \mathbf{p}_{n m}\left(r^{\prime}\right) \mathbf{p}(r) \frac{\cos h \eta_{n m} z^{\prime}}{\eta_{n m}}\right] d \tau^{\prime}\right]
\end{aligned}
$$$$
\begin{aligned}
\mathbf{E}_{T E} & =\mathbf{A}, \\
\mathbf{E}_{T M} & =\mathbf{E}_{1}+\mathbf{B}, \text { and } \\
\mathbf{E}_{\text {Total }} & =\mathbf{E}_{T E}+\mathbf{E}_{T M} .
\end{aligned}
$$

Thus the contribution to the field from $\mathbf{E}_{1}$ is exactly equal and opposite to part of the contribution arising from $\mathbf{B}$, so that the total field may now be divided into two parts (1) a transverse electric field, $\mathbf{E}_{T E}$, which corresponds at low frequencies to inductive coupling, and (2) a transverse magnetic field, $\mathbf{E}_{T M}$, which corresponds at low frequencies to capacitative coupling. Thus

where $\eta_{n m}=\sqrt{\lambda_{n m}^{2}-k^{2}}$

The field for $z<z^{\prime}$ is obtained by interchanging $z$ and $z^{\prime}$ in eq 22 and 23 .

It is noticed first that the various modes are either in phase or 180 degrees out of phase, which is, of course necessary, since we have neither resistive nor radiation losses. Also, the field does not decrease exponentially with $z$, but contains a factor, $\left(1 \pm e^{-2 \gamma(d-z)}\right)$ which is insignificant at small values of attentuation, but becomes appreciable as the receiving loop approaches the end of the cavity. If the attenuator were used in this way, its calibration in decibels would not be linear with variation of $z$. This nonlinearity can be avoided if the attenuator is constructed as illustrated in figure 1 . The exciting loop is fixed relative to a fixed end of the cavity, and the receiving loop is fixed to the other end which may slide within the cavity, varying its length, so that $d-z$ is a constant. Since 


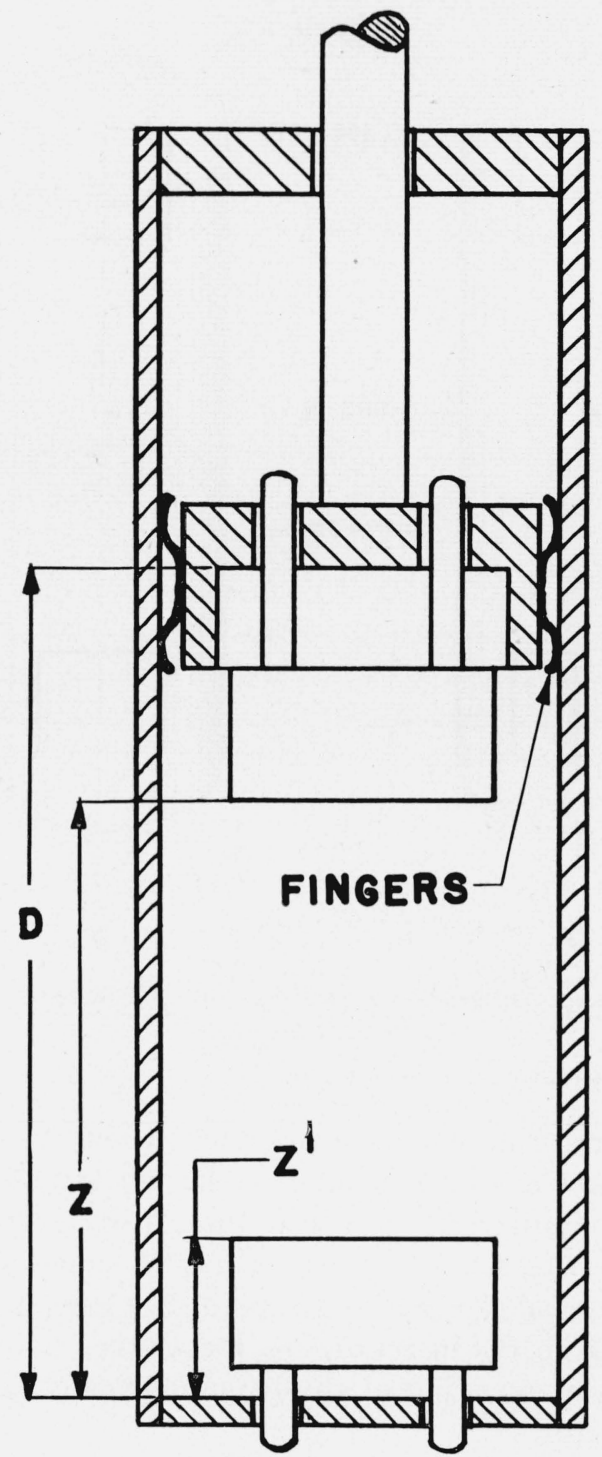

FIGURE 1.-Schematic drawing of attenuator.

$e^{-2 \gamma d} \ll 1$, the denominator is independent of $d$ and the ratio of $E_{T E}$ for a given mode, for two positions of the receiving loop, $z_{1}$ and $z_{2}$, is $e^{-\gamma\left(z_{1}-z_{2}\right)}$, and the nonlinearity is eliminated.

In general, an infinite number of modes are excited, so that the best one can do to insure purity of mode is to use symmetry of excitation which will not excite those unwanted modes with the lowest attenuation factor, and to use large enough values of $z$, such that the unwanted modes are sufficiently attentuated. For low enough frequency,

$$
\gamma_{n m}=\sqrt{\frac{v_{n m}^{2}}{a^{2}}-k^{2} \simeq} \frac{v_{n m}}{a}, \text { where } J_{n}^{\prime}\left(v_{n m}\right)=0 ;
$$

$$
\eta_{n m}=\sqrt{\frac{u_{n m}^{2}}{a^{2}}-k^{2} \simeq} \frac{u_{n m}}{a}, \text { where } J_{n}\left(u_{n m}\right)=0 .
$$

The first few values of $v_{n m}$ and $u_{n m}$ are as follows:

$$
\begin{array}{ll}
u_{01}=2.405 & v_{11}=1.841 \\
u_{11}=3.832 & v_{21}=3.05 \\
u_{21}=5.136 & v_{01}=3.832 \\
u_{02}=5.520 & v_{12}=5.33 \\
u_{12}=7.016 & v_{20}=7.016
\end{array}
$$

From the above values of $u_{n m}$ and $v_{n m}$ it is at once apparent that maximum purity of mode can be achieved by exciting the $T E_{11}$ mode, and eliminating the $T E_{01}, T E_{21}$, and $T M_{01}$ modes through symmetry considerations.

Consider the exciting current distribution in a plane perpendicular to the axis of the cylinder, figure 2. Assume that corresponding to every

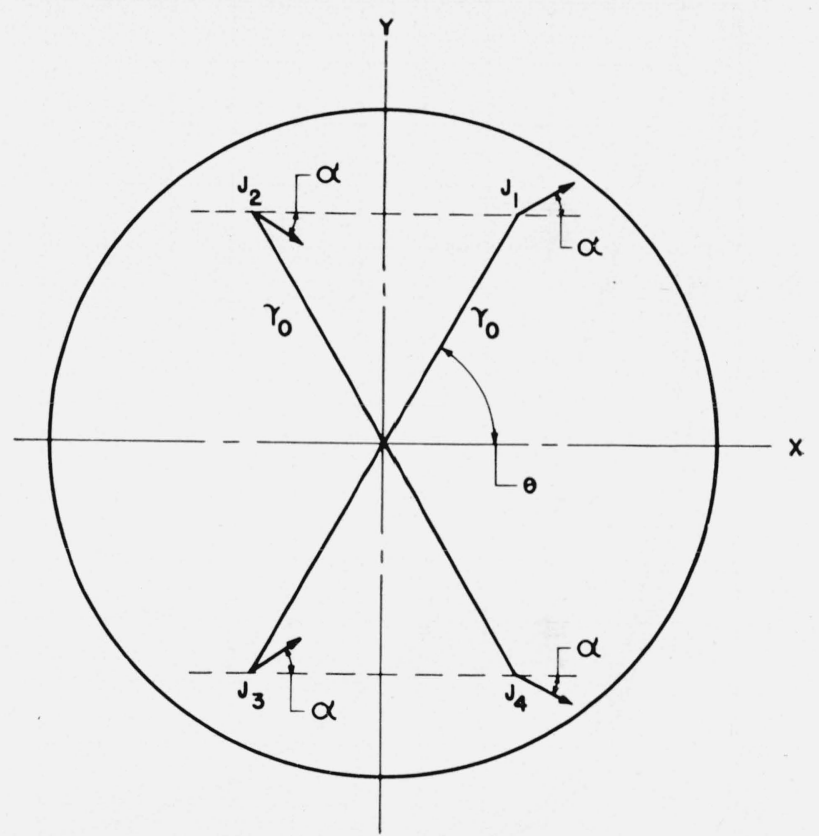

FIGURE 2.-Current distribution in plane perpendicular to axis of cylinder.

current element, $\mathbf{J}_{1}\left(r_{0}, \theta, \alpha\right)$, where $r_{0}$ is the distance of the element from the axis of the cylinder, $\theta$ is the angular displacement of its position vector with respect to the $x$-axis, and $\alpha$ is the angle between $\mathbf{J}$, and the $x$-axis, there exist corresponding current elements,

$\mathbf{J}_{2}\left(r_{0}, \pi-\theta,-\alpha\right), \mathbf{J}_{3}\left(r_{0}, \pi+\theta, \alpha\right), \mathbf{J}_{4}\left(r_{0}, 2 \pi-\theta,-\alpha\right)$.

Then, it immediately follows that

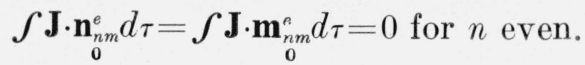


Further, if corresponding to a positive $z$-component of current at $\left(r_{0}, 2 \pi-\theta\right)$ and $\left(r_{0}, \theta\right)$, there exist equal negative $z$-components of current at $\left(r_{0}, \pi-\theta\right)$ and $\left(r_{0}, \pi+\theta\right)$, then $\int \mathbf{J} \cdot \mathbf{p}_{n m}^{e} d \tau=0$, for $n$ even.

In general, if the exciting current symmetry is such that the distribution for $x<0$ is the negative mirror image of that for $x>0$, and if the distribution for $y<0$ is the positive mirror image of that for $y>0$, then only those modes will be excited for which $n$ is odd.

A convenient index of attenuator performance is the percentage error made in a measurement of voltage as a function of the distance between exciting and receiving coils. The computed value

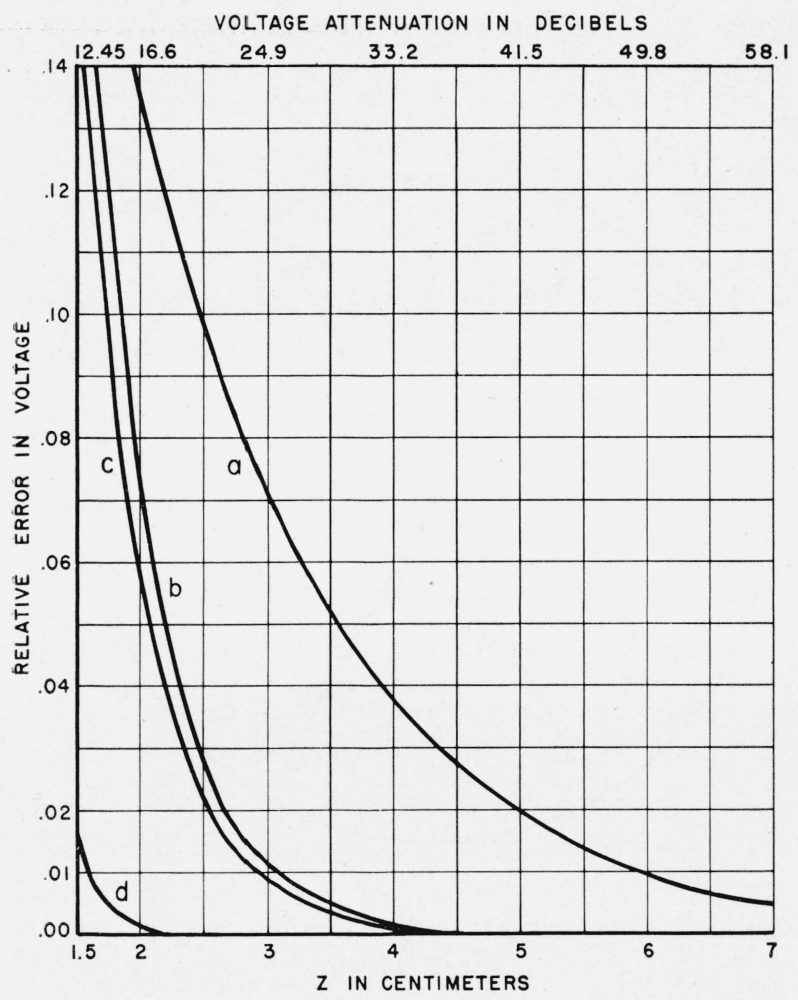

Figure 3.-Relative error in measurement of voltage due to the presence of unwanted modes.

a, Unsymmetric source in circular guide (fig. 5 b); b, symmetric source in circular guide (fig. 5a); c, symmetric source in rectangular guide (fig. 6a); d, symmetric source in rectangular guide chosen to eliminate $T E_{l s}$ modes (fig. 6b).

$$
\begin{aligned}
\mathbf{E}_{l m n}= & \sqrt{\frac{8}{a b c}} \frac{1}{\sqrt{\frac{l^{2}}{a^{2}}+\frac{m^{2}}{b^{2}}+\frac{n^{2}}{c^{2}}}}\left[\frac{l}{a} \cos \frac{l \pi x}{a} \sin \frac{m \pi y}{b} \sin \frac{n \pi z}{c} \mathbf{i}+\frac{m}{b} \sin \frac{l \pi x}{a} \cos \frac{m \pi y}{b} \sin \frac{n \pi z}{c} \mathbf{j}+\right. \\
& \left.\frac{n}{c} \sin \frac{l \pi x}{a} \sin \frac{m \pi y}{b} \cos \frac{n \pi z}{c} \mathbf{k}\right]
\end{aligned}
$$


The two independent normalized vector wave functions of zero divergence are

and

$$
\mathbf{M}_{l m n}=\sqrt{\frac{8}{a b c}} \frac{1}{\sqrt{\frac{l^{2}}{a^{2}}+\frac{m^{2}}{b^{2}}}}\left[-\frac{m}{b} \sin \frac{m \pi y}{b} \cos \frac{l \pi x}{a} \mathbf{i}+\frac{l}{a} \sin \frac{l \pi x}{a} \cos \frac{m \pi y}{b} \mathbf{j}\right] \sin \frac{n \pi z}{c},
$$

$$
\mathbf{N}_{l m n}=\sqrt{\frac{8}{a b c}} \frac{1}{\sqrt{\frac{l^{2}}{a^{2}}+\frac{m^{2}}{b^{2}}+\frac{n^{2}}{c^{2}}}} \frac{1}{\sqrt{\frac{l^{2}}{a^{2}}+\frac{m^{2}}{b^{2}}}} \frac{1}{\sqrt{1+\delta_{0}^{n}}}\left[\begin{array}{l}
-\frac{l n}{a c} \cos \frac{l \pi x}{a} \sin \frac{m \pi y}{b} \sin \frac{n \pi z}{c} \mathbf{i} \\
-\frac{m n}{b c} \sin \frac{l \pi x}{a} \cos \frac{m \pi y}{b} \sin \frac{n \pi z}{c} \mathbf{j} \\
+\left(\frac{l^{2}}{a^{2}}+\frac{m^{2}}{b^{2}}\right) \sin \frac{l \pi x}{a} \sin \frac{m \pi y}{b} \cos \frac{n \pi z}{c} \mathbf{k}
\end{array}\right]
$$

Again, when we solve for the field excited by a distribution of current, $\mathbf{J}\left(r^{\prime}\right)$, part of the field developed into $\mathbf{N}_{l m n}$ functions exactly cancels that part of the field whose curl is zero, so that the resultant field, E, may be expressed as the sum of transverse electric and transverse magnetic components.

$$
\begin{gathered}
\text { where } \mathbf{E}_{T E}=\frac{4 j \omega \mu}{a b} \boldsymbol{E} \mathbf{J}\left(r^{\prime}\right) \cdot \sum_{l m} \frac{\mathbf{P}_{l m}\left(r^{\prime}\right) \mathbf{P}_{l m}(r) \sin h \eta z^{\prime}}{\eta_{l m}\left(\frac{l^{2}}{a^{2}}+\frac{m^{2}}{b^{2}}\right)}\left[\frac{e^{-\eta z}}{1-e^{-2 \eta c}}\left(1-e^{-2 \eta(c-z)}\right)\right] d \tau^{\prime} \\
\mathbf{E}_{T M}=\frac{-4 j}{\omega \epsilon a b} \mathcal{J} \mathbf{J}\left(r^{\prime}\right) \cdot \sum_{l m} \frac{e^{-\eta z}}{1-e^{-\eta c}}\left[\left\{\frac{\mathbf{S}\left(r^{\prime}\right) \mathbf{S}(r) \eta \sin h \eta z^{\prime}}{\left(\frac{l^{2}}{a^{2}}+\frac{m^{2}}{b^{2}}\right)}+\pi \mathbf{Q}\left(r^{\prime}\right) \mathbf{S}(r) \cos h \eta z^{\prime}\right\}\left(1-e^{-2 \eta(c-z)}\right)-\right. \\
\left.\left\{\pi \mathbf{S}\left(r^{\prime}\right) \mathbf{Q}(r) \sin h \eta z^{\prime}+\pi^{2}\left(\frac{l^{2}}{a^{2}}+\frac{m^{2}}{b^{2}}\right) \mathbf{Q}\left(r^{\prime}\right) \frac{\mathbf{Q}(r) \cos h \eta z^{\prime}}{\eta}\right\}\left(1+e^{-2 \eta(c-z)}\right)\right] d \tau^{\prime} \\
\mathbf{P}_{l m}=\left[-\frac{m}{b} \sin \frac{m \pi y}{b} \cos \frac{l \pi x}{a} \mathbf{i}+\frac{l}{a} \sin \frac{l \pi x}{a} \cos \frac{m \pi y}{b} \mathbf{j}\right], \eta \eta_{l m}=\sqrt{\left(\frac{\pi l}{a}\right)^{2}+\left(\frac{\pi m}{b}\right)^{2}-k^{2}} \\
\mathbf{S}_{l m}=\left[\frac{l}{a} \cos \frac{l \pi x}{a} \sin \frac{m \pi y}{b} \mathbf{i}+\frac{m}{b} \sin \frac{l \pi x}{a} \cos \frac{m \pi y}{b} \mathbf{j}\right], \mathbf{Q}_{l m}=\sin \frac{l \pi x}{a} \sin \frac{m \pi y}{b} \mathbf{k},
\end{gathered}
$$

$r^{\prime}$ and $r$ refer respectively to the position coordinates of $\mathbf{J}$, and the point at which the field is evaluated.

It will be noticed that $\mathbf{E}_{T E}$ and $\mathbf{E}_{T M}$ have the same value of attentuation constant, for the same mode numbers, $l, m$.

Also, in line with our previous discussion, we shall assume that the receiving coil is rigidly fixed to the movable end of the cavity, so that the factor $\left(1 \pm e^{-2 \eta(c-z)}\right)$ is cancelled out in the measurement of the ratio of any two voltages. The magnitude of the error introduced by the factor $\left(1-e^{-2 \eta c}\right)$ is such, that if the smallest value of $c$ used is always greater than 1.5 times the larger dimension of cross section, the factor will introduce an error of less than 1 part in 10,000 for the $T E_{01}$ mode, and less for higher modes.

One advantage of an attenuator tube with rectangular cross section over that with circular cross section is that for the former, there is no need to discriminate between $T E$ and $T M$ type modes, for the same index numbers, $l, m$, since for given values of $l$ and $m$, the voltage induced in the collecting coil will vary as $e^{-\sqrt{\frac{\pi^{2} l^{2}}{a^{2}}+\frac{\pi^{2} m^{2}}{b^{2}}-k^{2} z}}$ for $T M$ and $T E$ type modes, alike. Of course, for tubes of circular cross section this is not true, except for cases of accidental degeneracy.

Furthermore, for tubes of rectangular cross section, it is possible to secure greater suppression of unwanted modes by a suitable choice of ratio of the two dimensions of cross section. The following table enumerates the ratios of attenuation 
constants for various modes to that of the lowest mode, for various ratios of rectangular cross section dimensions and for circular cross section.

It becomes apparent that the optimum practical ratio in the above table is $b / a=3$, and that this choice allows the unwanted modes to decay much faster than for circular cross section.

TABLE 1.-Ratio of attenuation constant of higher modes to that of the dominant mode

\begin{tabular}{|c|c|c|c|c|c|c|}
\hline \multicolumn{5}{|c|}{ Rectangular waveguide } & \multicolumn{2}{|c|}{ Circular waveguide } \\
\hline Mode & $\frac{\eta}{\eta_{T E_{01}}}$ & $\frac{\eta}{\eta_{T E_{01}}}$ & $\frac{\eta}{\eta_{T E_{01}}}$ & $\frac{\eta}{\eta_{T E_{01}}}$ & $\frac{\eta}{\eta_{T E_{11}}}$ & Mode \\
\hline & $a=b$ & $a=0.9 b$ & $a=\frac{b}{2}$ & $a=\frac{b}{3}$ & & \\
\hline$T E_{01 \ldots} \ldots \ldots$. & 1 & 1 & $i$ & 1 & 1 & $T E_{11}$ \\
\hline$T E_{02} \ldots \ldots \ldots$ & 2 & 2 & 2 & 2 & 1. 31 & $T M_{\mathrm{C1}}$ \\
\hline$T E_{03} \ldots \ldots \ldots$ & 3 & 3 & 3 & 3 & 1. 66 & $T E_{21}$ \\
\hline$T E_{10} \ldots \ldots \ldots$ & 1 & 1.1 & 2 & 3 & 2. 08 & $T E_{01}, T M_{11}$ \\
\hline$T E_{2 \mathrm{C}} \ldots \ldots \ldots \ldots$ & 2 & 2.2 & 4 & 6 & 2. 79 & $T M_{21}$ \\
\hline$T E_{11}, T M_{11} \ldots$. & 1.41 & 1.5 & 2. 2 & 3.1 & 2. 90 & $T E_{12}$ \\
\hline$T E_{21}, T M_{21} \ldots \ldots$ & 2. 2 & 2.4 & 41 & 6.1 & 3.0 & $T M M_{2}$ \\
\hline
\end{tabular}

If the exciting current distribution is chosen, so that to each element of current corresponds its negative mirror image with respect to the plane $x=\frac{a}{2}$, and its positive mirror image with respect to the plane $y=\frac{b}{2}$, then it is easily seen that

$$
\begin{aligned}
& \left.\begin{array}{l}
\boldsymbol{S} \mathbf{J}\left(r^{\prime}\right) \cdot \mathbf{P}_{l m} d \tau^{\prime} \\
\boldsymbol{S} \mathbf{J}\left(r^{\prime}\right) \cdot \mathbf{S}_{l m} d \tau^{\prime} \\
\boldsymbol{S} \mathbf{J}\left(r^{\prime}\right) \cdot \mathbf{Q}_{l m} d \tau^{\prime}
\end{array}\right\}=0 \text {, for } l \text { odd or } m \text { even. }
\end{aligned}
$$

The advantages of employing this type of symmetrical source distribution becomes apparent when we consult table 1 , and recognize that it eliminates four out of the first six spurious modes.

Another advantage of rectangular attenuators over those of circular cross section is the ease with which current distributions of slightly higher symmetry may be used to eliminate further spurious modes, by placing the current elements at the nodal points of the undesired modes. The relative voltage error for a rectangular attenuator with a simple symmetric loop source, and a double loop source, (fig. 5) whose position is so chosen that modes for which $m=3$ are not excited, is graphed in figure 3.
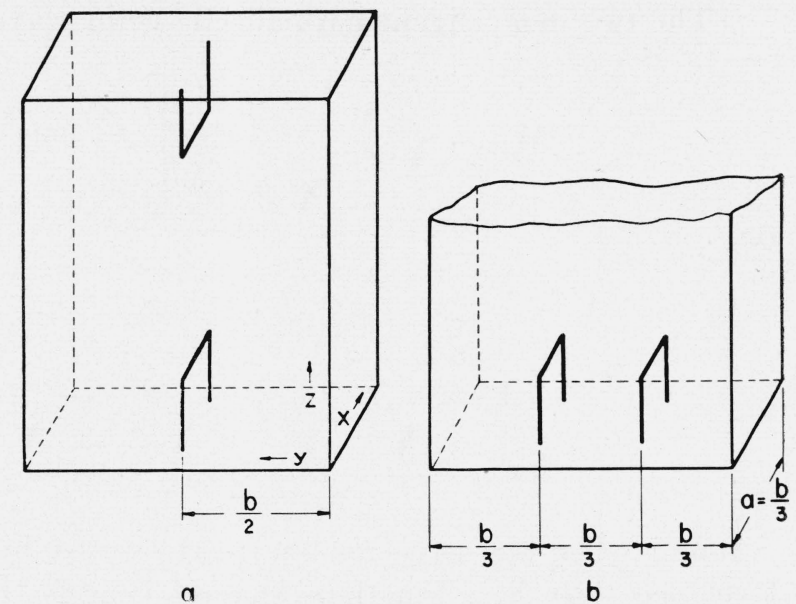

FIGURE 5.-Schematic of exciting and receiving loops in rectangular guide.

a, Symmetric loop; b, symmetric double loop placed at nodes so that no modes are excited, for which $m=3$.

\section{Conclusion}

It may be concluded, in general, that attenuators of rectangular cross section have two advantages over those of circular cross section:

1. Greater symmetry with respect to the dominant mode in the rectangular guide permit one to eliminate more spurious modes; 2 . a slight departure from symmetry in the rectangular guide is not so serious as in the circular guide, as the spurious modes decay more rapidly in the former.

The author expresses his thanks to Dr. Harold Lyons for his interest and helpful discussions during the course of this investigation.

\section{References}

[1] D. E. Harnett and N. P. Case, IRE 23578 (June 1935).

[2] Deutsche Mathematiker-Vereinigung Jahresbericht 21 309 (1912).

[3] W: Heitler, Quantum theory of radiation, p. 47 (Oxford Univ. Press, 1944).

[4] E. U. Condon, Rev. Mod. Phys. 14, 341 (Oct. 1942).

[5] J. A. Stratton, Electromagnetic theory, p. 465 (McGraw-Hill Book Co., New York, N. Y., 1941).

[6] J. A. Stratton, Electromagnetic theory, p. 392 (Mcgraw-Hill Book Co., New York, N. Y., 1941).

[7] T. M. McRobert, Functions of a complex variable, p. 115 (MacMillan Co., New York, N. Y., 1933). 


\section{Appendix I}

1 Let $S_{1}=\sum_{l=1}^{\infty} \frac{\sin \frac{l \pi z^{\prime}}{d} \sin \frac{l \pi z}{d}}{\gamma^{2}+\frac{l^{2} \pi^{2}}{d^{2}}}=\frac{d^{2}}{\pi^{2}} \sum_{l=1}^{\infty} \frac{\sin \frac{l \pi z^{\prime}}{d} \sin \frac{l \pi z}{d}}{\frac{d^{2} \gamma^{2}}{\pi^{2}}+l^{2}}$.

2. Let $\frac{d \gamma}{\pi}=\beta$.

3. $S_{1}=\frac{d^{2}}{2 \pi^{2}} \sum_{l=1}^{\infty} \frac{\cos \frac{l \pi}{d}\left(z-z^{\prime}\right)-\cos \frac{l \pi}{d}\left(z+z^{\prime}\right)}{\beta^{2}+l^{2}}=$

$$
\frac{d^{2}}{4 \pi^{2}} \sum_{l=-\infty}^{\infty} \frac{\cos \alpha l-\cos \alpha^{\prime} l}{\beta^{2}+l^{2}} .
$$

4. Where $\alpha=\frac{\pi\left(z-z^{\prime}\right)}{d}, \alpha^{\prime}=\frac{\pi\left(z+z^{\prime}\right)}{d}$.

5. Consider $f(z)=\frac{e^{j \alpha z}}{\left(\beta^{2}+z^{2}\right)\left(e^{2 \pi j z}-1\right)}$, let $z=R(\cos \theta+j \sin \theta)$. Then $\lim _{R \rightarrow \infty}|Z f(z)|=0$, and $\lim _{R \rightarrow \infty} z f(z)=0$, provided
$0 \leq \alpha \leq 2 \pi$.

Then, [7] the line integral of $f(z)$ around an infinite circle enclosing the complex plane vanishes.

$6 \lim _{R \rightarrow \infty} \oint f(z) d z=0$.

Since $f(z)$ has poles at $\pm j \beta, 0, \pm 1, \pm 2, \ldots$.

7. $0=\sum_{l=-\infty}^{l-\infty} \frac{e^{j \alpha l}}{\beta^{2}+l^{2}}+\frac{\pi}{\beta}\left\{\frac{e^{-\alpha \beta}}{e^{-2 \pi \beta}-1}-\frac{e^{\alpha \beta}}{e^{2 \pi \beta}-1}\right\}$.
8. or $\sum_{-\infty}^{\infty} \frac{\cos \alpha l}{\beta^{2}+l^{2}}=\frac{\pi}{\beta}\left[\frac{e^{\alpha \zeta}}{e^{2 \pi \beta}-1}-\frac{e^{-\alpha \beta}}{e^{-2 \pi \beta}-1}\right]$.

On substituting eq 8 in eq 3 , with a little algebra we get immediately,

9. $S_{1}=\frac{d}{2 \gamma} \frac{\sinh \gamma z^{\prime}\left(1-e^{-2 \gamma(d-z)}\right)}{1-e^{-2 \gamma d}} e^{-\gamma z}$, for $z>z^{\prime}$.

\section{Appendix II}

1. Let $S_{2}=\sum_{l=0}^{\infty} \frac{\frac{l \pi}{d} \sin \frac{l \pi z^{\prime}}{d} \cos \frac{l \pi z}{d}}{\gamma^{2}+\left(\frac{l \pi}{d}\right)^{2}}$.

2. Then $S_{2}=\frac{d S_{1}}{d z}=\frac{d}{-2} \sinh \gamma z^{\prime}\left[\frac{e^{\gamma} z}{e^{2 \gamma d}-1}-\frac{e^{-\gamma} z}{e^{-2 \gamma d}-1}\right]$.

3. Let $S_{3}=\sum_{l=1}^{\infty} \frac{\frac{l \pi}{d} \cos \frac{l \pi z^{\prime}}{d} \sin \frac{l \pi z}{d}}{\gamma^{2}+\left(\frac{l \pi}{d}\right)^{2}}$.

4. Then $S_{3}=\frac{d S_{1}}{d z^{\prime}}=\frac{-d}{2} \cosh \gamma z^{\prime}\left[\frac{e^{\gamma z^{\prime}}}{e^{2 \gamma d}-1}+\frac{e^{-\gamma z}}{e^{-2 \gamma d}-1}\right]$.

5. Let $S_{4}=\sum_{0}^{\infty} \frac{\left(\frac{l \pi}{d}\right)^{2} \cos \frac{l \pi z}{d} \cos \frac{l \pi z^{\prime}}{d}}{\gamma^{2}+\left(\frac{l \pi}{d}\right)^{2}}$.

6. Then $S_{4}=\frac{d S_{2}}{d z^{\prime}}=\frac{-\gamma d}{2} \cosh \gamma z^{\prime}\left[\frac{e^{\gamma z}}{e^{2 \gamma d}-1}-\frac{e^{-\gamma z}}{e^{-2 \gamma d}-1}\right]$

Washington, September 23, 1946. 